\title{
A Case of Incidentally Detected Urothelial Carcinoma of Renal Pelvis
}

\author{
(D) Anoop Handa1, (1) Sharat Chandra Dash1, (D) Gagandeep Singh1, (1) Nimit Solanki2, (1) Kunwara Vishal Singh1, \\ (1) Gaurav Pratap Singh Gahlot ${ }^{3}$ \\ ${ }^{1}$ Army Hospital Research and Referral, Clinic of Urology, New Delhi, India \\ 2Base Hospital, Clinic of Urology, New Delhi, India \\ 3 Western Command Hospital, Clinic of Pathology, Chandimandir, Haryana, India
}

\begin{abstract}
Urothelial carcinomas of the renal pelvis are rare malignancies presenting commonly in older individuals and are characterised by flank pain, haematuria and palpable mass. Squamous differentiation is a common urothelial carcinoma and is usually associated with factors causing chronic irritation such as renal stone, infection and inflammation. We report a case of a young male patient who was incidentally diagnosed with right pelvic-ureteric junction obstruction during evaluation for Hodgkin lymphoma. After completion of chemotherapy for Hodgkin lymphoma, the patient underwent laparoscopic Anderson-Hynes pyeloplasty. The resected adynamic segment part of the pelvic-ureteric junction was sent for histopathological examination, which showed high-grade urothelial carcinoma with extensive squamous metaplasia. The patient was further managed with laparoscopic radical nephroureterectomy and lymphadenectomy. Our report is the first case of an incidental histologically detected urothelial carcinoma in a young patient without any radiological mass lesion.
\end{abstract}

Keywords: Urothelial carcinoma, pelvic-ureteric junction obstruction, squamous metaplasia, renal pelvis, pyeloplasty

\section{Introduction}

Upper urinary tract tumours involving the renal pelvis and ureter are relatively rare and seen in less than $5 \%$ of renal tumours. In a study, renal pelvic tumours accounted for $11.1 \%$ of all tumours, with the majority of urothelial origin (1). We report a rare case of urothelial carcinoma with extensive squamous metaplasia presenting as pelvic-ureteric junction obstruction that was diagnosed in the resected stenotic segment during AndersonHynes pyeloplasty. This histological diagnosis determined in the absence of typical symptoms or radiological mass lesions is notable from a clinical standpoint.

\section{Case Presentation}

A 28-year-old male, non-smoker, driver by occupation, presented with complaints of breathlessness, cough and 1-month weight loss. He had no history of abdominal pain, fever, haematuria or lower urinary tract symptoms. General and systemic physical examination were unremarkable. Routine haematological, biochemical and urinalysis findings were normal. Contrast-enhanced computed tomography (CT) chest and whole-body positron emission tomography (PET) scan revealed fluorodeoxyglucose (FDG) avid level IV cervical lymph nodes, mediastinal lymph nodes and gross dilatation of the right renal pelvis with cortical thinning $(5-8 \mathrm{~mm})$; the normal ureter was diagnosed with pelvic-ureteric junction obstruction (Figure 1). No FDG lesion was noted in the right pelvis, ureter and hilar region. Excisional biopsy of the right supraclavicular lymph node showed nodular sclerosis Hodgkin lymphoma (stage III). The patient was started on chemotherapy for Hodgkin
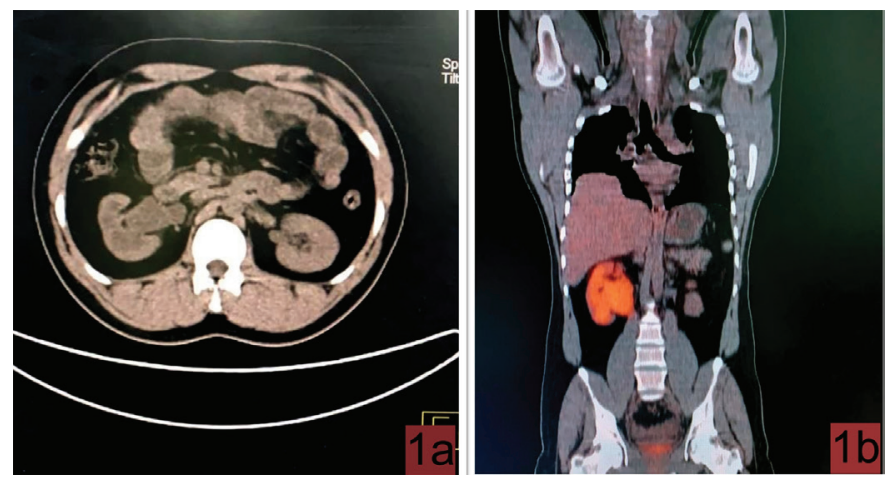

Figure 1. 1a. Axial section of computed tomography scan showing dilated right renal pelvis with parenchymal thinning. 1b. Coronal section of positron emission tomography scan showing physiological uptake in the right renal pelvis

Correspondence: Anoop Handa MD, Army Hospital Research and Referral, Clinic of Urology, New Delhi, India Phone: +7837035656Ｅ-mail: dr.anoop10@gmail.com ORCID-ID: orcid.org/0000-0003-2364-7854 Received: $16.08 .2020 \quad$ Accepted: 30.12 .2020

Cite this article as: Handa A, Dash SC, Singh G, Solanki N, Singh KV, Gahlot GPS. A Case of Incidentally Detected Urothelial Carcinoma of Renal Pelvis. J Urol 2021;8(1):62-64.

๑Copyright 2020 by the Association of Urological Surgery / Journal of Urological Surgery published by Galenos Publishing House. 
lymphoma; after completion of six sessions of adriamycin, bleomycin sulphate, vinblastine sulphate and dacarbazine chemotherapy, he was referred for obstruction management. Diethylenetriaminepentacetate scan revealed type 2 obstructive renogram curve with $\mathrm{T} 1 / 2$ of right kidney $26 \mathrm{~min}$, glomerular filtration rate of $26.4 \mathrm{~mL} / \mathrm{min}$ and split function of $35 \%$. The patient underwent laparoscopic Anderson-Hynes dismembered pyeloplasty. Intraoperatively, large dilated pelvis was seen with tapering at the pelvic-ureteric junction, the stenotic segment was resected at approximately $1 \mathrm{~cm}$, and reduction pyeloplasty was performed. Crossing vessels, peri-pelvic adhesions, or enlarged lymph nodes were not noted. No growth or any abnormal area was observed in the pelvis. Histopathology of the specimen showed high-grade transitional cell carcinoma with extensive squamous metaplasia. The underlying stroma showed extensive infiltrate by atypical squamous cells. Focal keratinisation was identified, and on immunohistochemistry, both p63 and GATA3 were positive in neoplastic cells (Figure 2). The patient then underwent laparoscopic radical nephroureterectomy with excision of the bladder cuff with lymph node dissection. No growth or abnormal area was visible in gross specimen. Histological examination revealed urothelial lining high-grade dysplasia at the pelvic-ureteric junction, with no lymph node invasion. The post-operative period was uneventful. The patient was asymptomatic during the 6-month follow-up, with no evidence of radiological or biochemical recurrence. Cystoscopy findings of the lower urinary tract were normal.

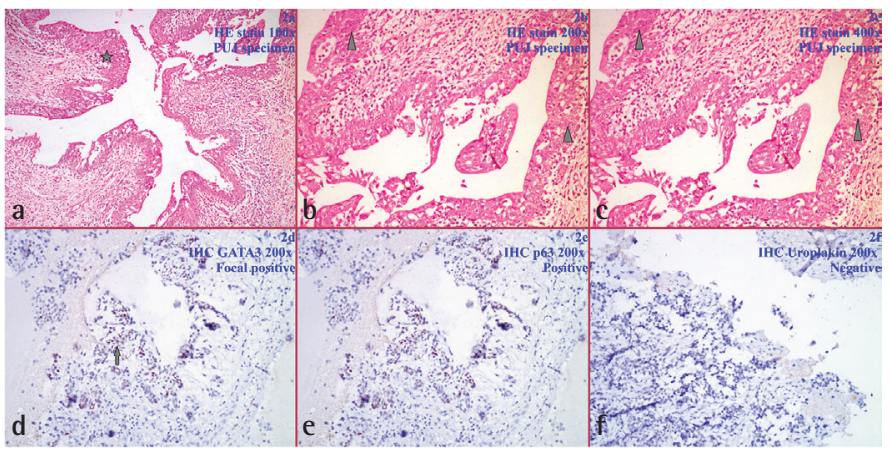

Figure 2. Microphotographs of Haematoxylin-Eosin stained sections of pelvic-ureteric junction lesion show urothelial lining (star 2a;100x) with foci of squamous differentiation (arrowhead 2b;200x, 2c;400x). Tumour cells show immunopositivity for GATA3 (arrow 2d; 200x) and P63 (arrow 2e; 200x) and immunonegativity for uroplakin $(2 \mathrm{f} ; 200 \mathrm{x})$

\section{Discussion}

Urothelial carcinoma with squamous differentiation of the renal pelvis are rare malignancies of the upper urinary tract. The common causes for squamous metaplasia in the urothelium are long-standing renal calculi, chronic analgesic abuse, chemicals, vitamin A deficiency, smoking, schistosomiasis and radiotherapy (2).
Urothelial tumours of the renal pelvis are most common in older individuals aged 60-65 years. Men are mostly affected, accounting for $>70 \%$ of cases. Most patients present with pain due to local extension or pelvic-ureteric junction obstruction, palpable mass and haematuria. Paraneoplastic syndromes such as hypercalcaemia and thrombocytosis may be associated in few cases. Radiological findings include a solid infiltrating mass with hydronephrosis, with or without calcifications (3).

Cases of squamous metaplasia associated with primary pelvicureteric junction obstruction have been reported; however, in most of them, a radiological mass lesion was detected or was non-functional due to large hydronephrosis $(4,5)$. In our case, the patient had no risk factors associated with urothelial carcinoma and no radiological mass lesion was seen on CT scan or any FDG avid lesion on PET scan. Gross inspection of the renal pelvis did not show any mass lesion or calcification.

This histopathological diagnosis remains a diagnostic challenge. True squamous differentiation requires evidence of keratinisation, intercellular bridges, or both on routine haematoxylin-eosin staining (6). There are certain immunohistochemical differences between these areas and a conventional urothelial tumour component. In this case, focal areas of keratinisation were present and GATA-3 stain highlighted the presence of neoplastic urothelial cells.

The standard treatment for these tumours is surgery. Radiotherapy/chemotherapy provides marginal benefits only. Radical nephroureterectomy with excision of the bladder cuff is the treatment of choice in these patients (7).

We report a rare incidental diagnosis of urothelial carcinoma with extensive squamous differentiation in resected stenotic segment of pelvic-ureteric junction obstruction in a young patient with no risk factors. This case report highlights the significance of histopathological examination of resected stenotic segment and need for meticulous sampling of the renal pelvis by the pathologist in such specimens.

\section{Ethics}

Informed Consent: No specific patient photographs were used in this manuscript, so no informed consent for publication was taken.

Peer-review: Externally and internally peer-reviewed.

\section{Authorship Contributions}

Surgical and Medical Practices: S.C.D., N.S., Concept: A.H., G.S., Design: A.H., G.S., Data Collection or Processing: N.S., K.V.S., G.P.S.G., Analysis or Interpretation: S.C.D., Literature Search: A.H., G.S., K.V.S., Writing: A.H., S.C.D., G.S., G.P.S.G. 
Conflict of Interest: No conflict of interest was declared by the authors.

Financial Disclosure: The authors declared that this study received no financial support.

\section{References}

1. Kini $H$, Sridevi $H B$, Suresh $P K$, Guni $L P$, Bhat $S$, Kini JR. Spectrum of Lesions Affecting the Renal Pelvis and Pelviureteric Junction: A 13-Year Retrospective Analysis. J Clin Diagn Res 2016;10:EC01-4.

2. Holmang $\mathrm{S}$, Lele SM, Johansson SL. Squamous cell carcinoma of the renal pelvis and ureter: incidence, symptoms, treatment and outcome. J Urol 2007;178:51-56.
3. Singh V, Sinha RJ, Sankhwar SN, Mehrotra B, Ahmed N, Mehrotra S. Squamous cell carcinoma of the kidney - rarity redefined: Case series with review of literature. J Cancer Sci Ther 2010;2:82-85.

4. Vasudeva P, Kumar N, Kumar A. Transitional cell carcinoma: A rare development in congenital ureteropelvic junction obstruction kidney. Indian J Urol 2016;32:79-80.

5. Gaur K, Gupta L, Saran RK, Ghuliani D. Staging a great escape -Incidentally detected renal urothelial carcinoma with extensive squamous metaplasia presenting as pyonephrosis. J Can Res Ther 2019;15:159-162.

6. Zhai 0 J, Black J, Ayala AG, Ro JY. Histologic variants of infiltrating urothelial carcinoma. Arch Pathol Lab Med 2007;131:1244-1256.

7. Kirkali Z, Tuzel E. Transitional cell carcinoma of the ureter and renal pelvis. Crit Rev Oncol Hematol 2003;47:155-169. 